\title{
Optimization Intensive Energy Harvesting
}

\author{
Mahsan Rofouei, Mohammad Ali Ghodrat, Miodrag Potkonjak \\ Computer Science Department, \\ University of California, Los Angeles (UCLA) \\ Los Angeles, CA, USA \\ \{mahsan, ghodrat, miodrag\}@cs.ucla.edu
}

\author{
Alfonso Martinez-Nova \\ Nursing Department \\ University of Extremadura \\ Plasencia, CC, Spain \\ podoalf@unex.es
}

\begin{abstract}
Instrumented Medical Shoes (MSs) are equipped with a variety of sensors for measurement of quantities such as pressure, acceleration, and temperature which are often greatly beneficial in numerous diagnosis, monitoring, rehabilitation, and other medical tasks. One of primary limiting factors of MSs is their energy sensitivity. In order to overcome this limitation, we have developed an optimization intensive approach for energy harvesting. Our goal is to size and position a single piezoelectric transducer for energy generation in a medical shoe in such a way that maximal energy is collected and/or specified maximal voltage is achieved while collecting energy. We propose a scenario approach that provides statistically sound solution and evaluate our approach using our medical shoe simulator for subject specific energy harvesting and generic MS scavenging. We could get $3.7 \mathrm{X}$ energy gain compare to smallest size sensor and 1.3X energy gain compared to sensor with the size of a shoe.
\end{abstract}

Keywords-energy harvesting; medical shoes;

\section{INTRODUCTION}

Sensor-based medical equipment is on a brink of revolutionizing many tasks in diagnosis, tracking, treatment, rehabilitation and many other medical activities $[16,17]$. These systems provide unprecedented comprehensive and real-time data collection with high convenience and reliability. However, there are several major obstacles for their more widespread application. Among them, energy supply is one of the most acute constraints. In a sense, energy harvesting is an ultimate solution. Unfortunately, the current generation of energy harvesters is rarely capable of collecting adequate amount of energy and providing sufficient power.

We focus on energy harvesting in medical shoes with an objective to create and position a single mechanical piezoelectric harvester in such a way that we collect the maximal amount of energy and/or maximize the highest available energy while specified maximal voltage is achieved. The backbone of the approach can be summarized in the following way: We first analyze the pressure sensed at large number of points in the medical shoe during walking trails. Both the collected energy and the maximal achievable power are functions of the pressure. The key trade-off is that high pressure at a particular moment enables higher total energy if collected. This value is the function of the maximal pressure. However, in order to collect a large amount of energy it is not only sufficient to aim for a high pressure; we also need a large force, i.e. relatively large force over relatively large area. Therefore, it is essential to select size and location of a piezoelectric energy harvester to achieve a quality trade-off between collected energy and maximal collected energy and hence, maximal achievable voltage.

In addition to the first quantitative and optimization intensive treatment of energy harvesting in MSs our main contribution is a new algorithm for optimization under uncertainty. The algorithm is named scenario-based because it uses a combination of statistical and combinatorial optimization techniques to find and evaluate the maximal energy harvesting problem in sensor shoes. The key idea is to select a small number of time samples and conduct optimization using only these samples. Once the solution is generated, we evaluate it by calculating the interval of confidence within learn-and-test or re-sampling paradigm. The approach is also applicable to a customized shoe for a particular subject as well as its generic version. All results are obtained using data from BioFoot medical shoe[7].

The remainder of the paper is organized as follows: In Section II a description of related work is provided. Next, in Section III preliminaries for this paper are described. The basis of our proposed scenario-based algorithm is described in Section IV. Following it in Section V, simulation results on applying the mentioned algorithm on data from 30 subjects are provided. Finally, conclusions are drawn in Section VI.

\section{RELATED WORK}

In the last two decades a large variety of medical shoes with a variety of sensors and interfaces have been designed and evaluated [4, 12]. Recently, they have been used in conjunction with artificial intelligence techniques for a variety of tasks ranging from event detection to new types of user interfaces (e.g. computer mouse) [10, 13]. More importantly, they have proven invaluable in many medical tasks [11]. There is surprisingly few efforts that report quantitative design and optimization of medical shoes [19]. Our main novelty is that we use sensing data and sensor location not for data collection but for optimized energy harvesting.

During the same period, energy and power emerged as premier design and operational metrics for many systems. A very attractive way to solve energy supply problem is to harvest (scavenge) environmental energy [1-3]. Numerous approaches emerged ranging from photovoltaic and vibration to human activity-based techniques to use of environmental electro-magnetic and nano-scale sources $[5,6,9]$. In [18] the authors have investigated power-harvesting from running shoes for generating power for wearable electronics. 


\section{PRELIMINARIES}

In this Section we review preliminaries covering information on medical shoes, specifically BioFoot [7] which we use for collecting samples. We also present information on energy harvesting and battery and capacitor models.

\section{A. Medical Shoes and Datasets}

With the advent of micro-system technologies, in-shoe plantar pressure measurement systems have become feasible. These systems are currently employed by clinicians and researchers to assess dynamic plantar pressures. TekScan FScan ${ }^{\circledR}$, Novel Pedar ${ }^{\circledR}$ and IVB Biofoot $\AA$ are examples of such systems [7, 14, 15]. For this study we used plantar pressure measurements from BioFoot ${ }^{\circledR}$ (IBV [7]). The system consists of two flexible, $0.7 \mathrm{~mm}$ insoles. Each insole has 64 small size piezoelectric sensors and has a round $5 \mathrm{~mm}^{2}$ diameter shape. The output signals are amplified and sent through the wireless transmitter attached to the subject's waist. The system allows measurements in $200 \mathrm{~m}$ of distance, therefore subjects can freely move around. The sampling rate for our measurements is $100 \mathrm{~Hz}$. Figure 1.a shows the BioFoot shoe.

\section{B. Energy Harvesting Model}

Electrical power can be harvested using multiple conversion mechanisms such as thermoelectric, electromagnetic and piezoelectric. Energy harvesting using piezoelectric conversion mechanism converts mechanical strain into electric current or voltage. This process can be described with charge displacement and can be modeled by a linear consecutive equation. According to [8], the energy stored in a parallel plate capacitor and corresponding voltage across piezoelectric $(V S)$ can be expressed as equations (1) and (2) respectively:

$$
W_{\text {out }}=\frac{1}{2}\left(\frac{\varepsilon_{0} \varepsilon_{r} A}{T}\right) V_{s}^{2} \quad(1), \quad V_{s}=\left(\frac{k_{33} F T}{A}\right) \sqrt{\frac{1}{Y \varepsilon_{0} \varepsilon_{r}}}
$$

In these equations, $\varepsilon_{0}$ and $\varepsilon_{r}$ represent free space permittivity and relative permittivity $(8.85 \mathrm{e}-12,200$ to 1350$)$ respectively. In addition, $T$ is thickness (191 um), $K_{33}$ is Electromechanical coupling coefficient (0.69) and $Y$ is Young's modulus $(63 \mathrm{GPa})$. Cross-sectional area of the piezoelectric material and applied force are represented by $A$ and $F$ respectively. Equations (1) and (2) show that the generated voltage is proportional to the amount of pressure applied $(F / A)$ while the generated energy is proportional to $F^{2} / A$.

\section{Battery and Capacitor Models}

The instantaneous power generated using piezoelectric harvesters might be relatively small depending on the amount of energy required to perform certain tasks, such as wireless transmission. Therefore it is necessary to somehow aggregate the energy before using it, either using capacitors or batteries. Recently there have been several attempts in designing power harvesting circuits. Figure 1.b shows a simplified power harvesting circuit [5]. As shown in the figure, in order to aggregate energy continuously the generated voltage needs to get higher as more energy is accumulated. One solution for this is to use multiple small batteries and charging each battery separately. Using a single battery, due to cost consideration requires intelligent energy harvesting mechanisms that produce high voltages.

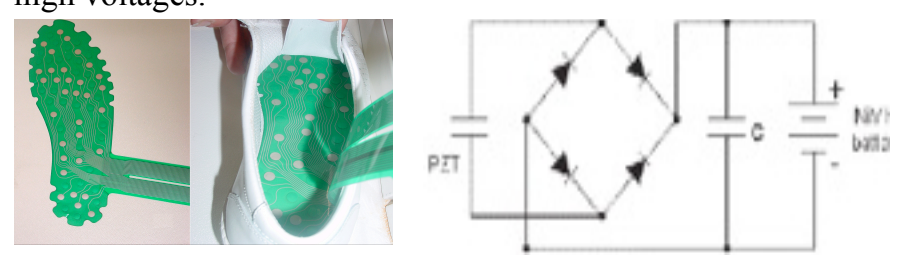

Figure 1. (a) BioFoot. (b) Schematic of Energy Harvesting Circuit [5].

\section{ENERGY HARVESTING}

In this Section, we formulate the problem of Maximal Energy Harvesting (MEH) and show that this problem is NPComplete by transforming maximal clique problem. We then propose our scenario approach for this problem in Part C.

\section{A. Problem Formulation}

In order to design self-sustainable shoe systems, careful examination of pressure distribution during movement is needed. We use the data from plantar pressure distribution of subjects during walking trails, to find best location to place energy harvesters. Due to cost considerations, we only look at a single harvester placement problem and find the location and size of the harvester. Therefore, the $\mathrm{MEH}$ problem can be defined as selection of shape/size of a single energy harvester in such a way that the amount of generated energy is optimized. The problem can be viewed from another perspective. When considering a case where restoring energy for later burst use is needed, the goal is to optimize for energy optimize for energy considering produced voltage levels.

We solve the MEH problem for two platforms: customized and generic. People have different walking patterns; therefore in order to maximize the amount of energy generated, customized platforms can be developed for individuals by studying their walking patterns. Figure 2 shows maximum pressure of different locations during walking, in four different individuals, where black represents maximum pressure. These images show how the pressure distribution is different among individuals; which in turn effects the decision of the location of energy harvester. Generic platforms can be also developed by considering most common features in different people.
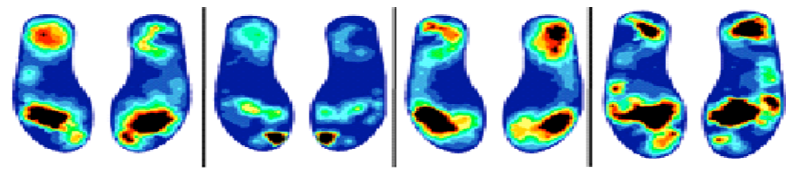

Figure 2. Maximum Pressure Distribution in Different Subjects

\section{B. Problem Complexity}

At the intuitive level the MEH problem may be defined as selection of piezoelectric material shape and size so that total collected energy is maximized. There are two primary sources of difficulty in solving this problem. The first is its statistical non-determinism and the second is its combinatorial structure. The first problem is due to uncertainty about future actions of the subject. Although the former is dominant in practice, the latter is a formidable obstacle since it is associated with solving a computational intractable problem. We can formulate a very simplified version of the MEH problem in the following way: 


\section{Problem: Deterministic Maximal Energy Harvesting (DMEH)}

Given: An undirected graph $G(V, E)$ with a set of vertices and edges, time slots $T_{1}, \ldots, T_{n}$ and pressure $P_{i, j}$ at each vertex $i$ for each $T_{j}$.

Objective and constraints: Select a set of time slots $T_{t}$ and vertices so that the total sum of pressures is maximized and in all selected time slots the pressure is at least $P$.

In order to prove that the DMEH problem is NP-complete we transform an arbitrary instance of one of 21 initial problems, maximal clique, into DMEH. We start with a graph $G_{G}$ which has the same set of vertices $V$ as graph $G$. Next, for each edge between vertices $V_{p}$ and $V_{q}$ in $G_{G}$, we create a time slot in DMEH where only corresponding vertices have pressure $P$ and all other vertices have zero pressure. Now, it is easy to see that if and only if graph $G_{G}$ has a clique of size $K$, we can collect total energy corresponding to sum of pressures $K * P$.

\section{Scenario Approach}

We use a scenario-based approach to find the best location and size of an energy harvester. The proposed algorithm uses a combination of statistical and combinatorial optimization techniques to find and evaluate the maximal energy harvester. The scenario approach uses walking samples from individuals. A small number of samples are chosen as input to a constructive iterative optimizer which finds the best location for the harvesters. This solution is evaluated through calculating the interval of confidence within a learn-and-test paradigm and more experiments are conducted if necessary. Figure 3 shows the flow of the scenario approach.

The algorithm starts with randomly choosing a small subset of samples of multiple people. Specifically, $K$ randomly selected samples are used as input to our constructive iterative optimizer (Algorithm II). The optimizer results in a single location for the placement of an energy harvester. We run the optimizer $m$ times, where $m$ is the number of scenarios (Algorithm $I$, lines 4-7), each time with $K$ random samples and build an interval of confidence for the solution of the optimizer. If the interval of confidence is too small to accept the solution, we repeat the above process, by increasing the number of samples: that is with a larger $K$ (Algorithm I, line 9). The above steps are repeated until an acceptable interval of confidence is found (Algorithm I, line 3). The solution of the constructive iterative optimizer at this step is presented as the final solution of the placement of a harvester.

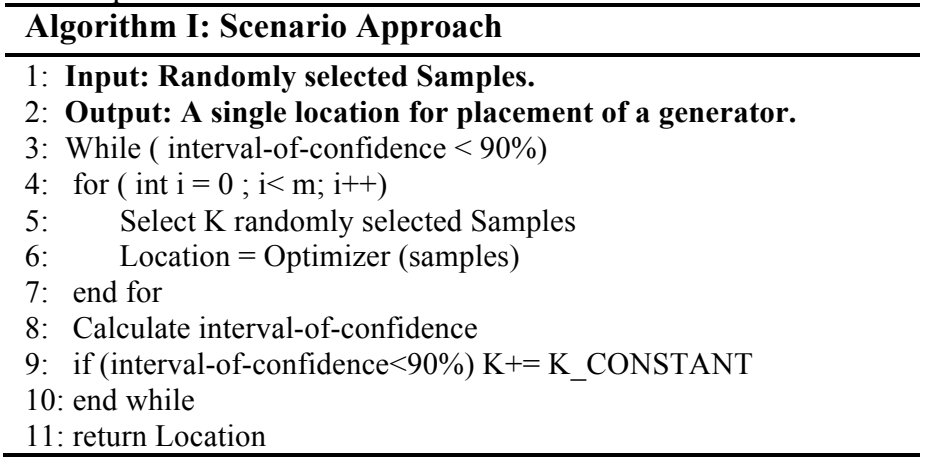

Figure 3. Algorithm I for Scenario approach
The constructive iterative optimizer (Algorithm II) works as follows: It starts with a single location $l_{i}$ to place a harvester. It checks to see if it satisfies problem constraints. If yes, it accepts it as a solution. In the next step it forms another solution called $l_{i^{\prime}}$ by combining $l_{i}$ with one of its geometric neighboring locations. It then decides to choose $l_{i}+1=l_{i}$ or $l_{i}+1=l_{i^{\prime}}$ based on the solution that optimizes the objective. These steps are performed until all locations have been explored. In Figure 4, Algorithm II describes the details of the constructive iterative optimizer. It starts with the smallest location generating the highest energy. Next, neighbor locations that can be merged to give a better energy value and satisfy voltage constraints are merged (lines 5-8 in Algorithm II). Then the merged location is added to the potential result list (line 9 in Algorithm II). This process is continued until there is no merging possibility. Finally the location with highest energy is returned.

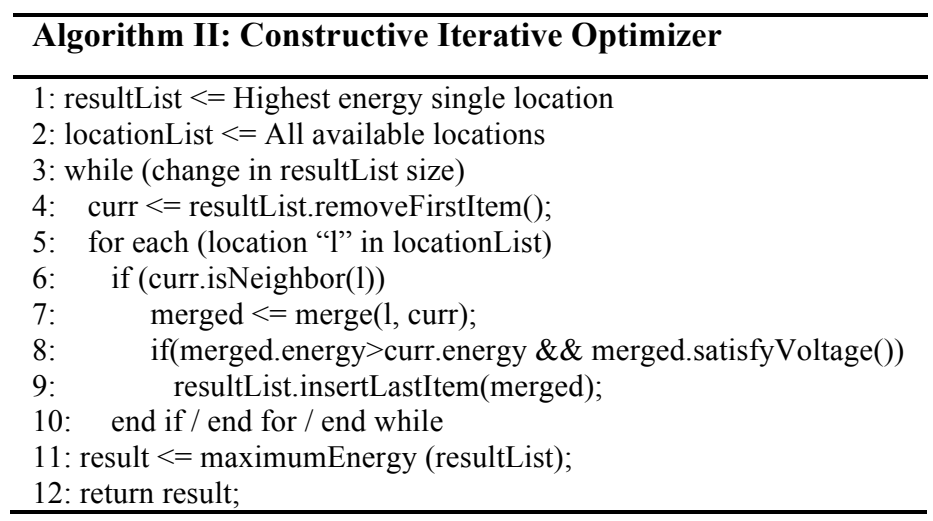

Figure 4. Algorithm II for Constructive Iterative Optimizer

As shown in Figure 1.b, in order to aggregate energy continuously, a relatively high generated voltage is needed to be able to accumulate energy. Therefore optimizing only for energy is not sufficient as a certain voltage level needs to be met to be able to store energy. The amount of voltage required is dependent upon many factors such as the amount of energy stored and the amount of energy consumed. Therefore in our approach we use a voltage/energy pareto technique, where we optimize for energy considering satisfying voltage requirements. Specifically, we consider a voltage level and require the voltage generated to meet this level by a percentage at all times. We use the same scenario technique described to find the best location of harvester to satisfy each voltage level.

\section{Simulation Results}

In this Section we present experimental results on using the described scenario approach. We use walking samples from 30 individuals to find the position for placing a single generator that would maximize the amount of energy generated and stored. The data is 6 seconds long for each individual. Here, due to space limitations, we only show the data from 5 users.

\section{A. Optimizing for Energy with Different Generator Sizes}

Based on our proposed approach the amount of energy consumed per second in a generic platform, optimizing just for 


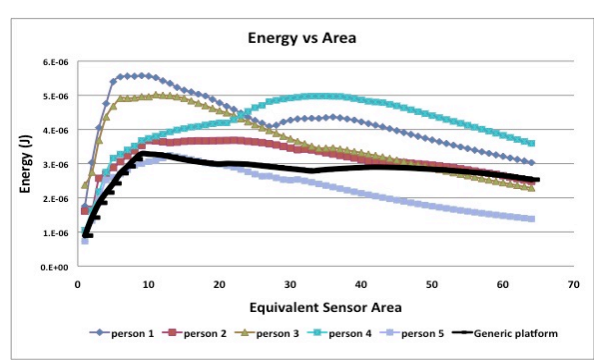

Figure 5. Energy vs. Generator area, Customized and Generic Platforms.

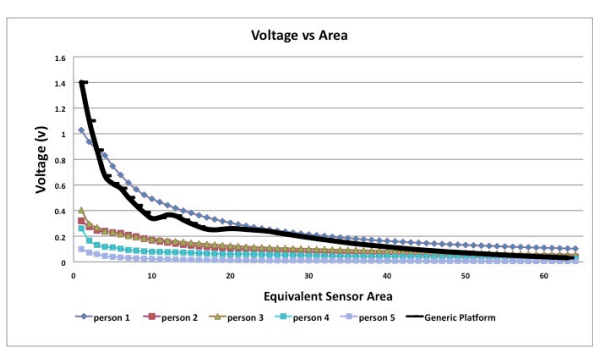

Figure 6. Voltage vs Generator area, Customized and Generic Platforms.

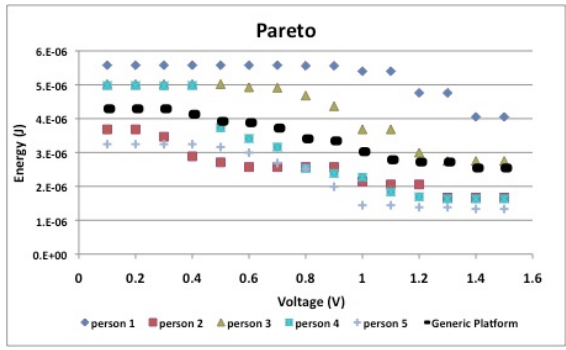

Figure 7. Paret vs Generator area, Customized and Generic Platforms. energy is $3.29 \mu \mathrm{J}$. To show the effectiveness and accuracy of our approach, we compare our results with two methods: 1) choosing smallest size generator at the location of maximum pressure $(0.89 \mu \mathrm{J})$ and 2$)$ choosing a large generator to cover all the locations that sense pressure $(2.53 \mu \mathrm{J})$. The results show that choosing a large area piezoelectric to cover all points or a small area piezoelectric at the position of maximum pressure are not correct choices. The interval of confidence for optimizing for energy is $(3.29 \pm 0.15 \mu \mathrm{J})$ with probability of $91 \%$.

Figure 5 shows the maximal amount of energy generated per second with different generator areas in both a customized and generic platform. As shown, if the goal is to only maximize the amount of energy generated, a generator with equivalent sensor area of 12 should be chosen for the generic platform. We also show customized results for 5 of the individuals.

\section{B. Optimizing for Voltage with Different Generator Sizes}

Figure 5 showed the maximal amount of energy generated per second with different size generators. However as discussed in Section IV, high voltages are also desired when generating energy, specifically for storing energy. Figure 6 shows the maximal amount of voltage generated per second in different generator sizes for the same subjects in Figure 5. As expected, according to equation (2), smaller area generators generate more voltage. With the increase in generator area, the amount of voltage generated becomes smaller.

\section{Optimal Energy-Volage Points}

According to Figure 1.b, in order to aggregate energy continuously, the generated voltage needs to be relatively high as more energy is accumulated. Therefore optimizing just for energy or just for voltage is insufficient. We use a voltage/energy pareto technique, where we optimize for energy while at the same time satisfying specific voltage requirements.

We consider a specific voltage level and require the voltage generated to satisfy this level by a specific percentage at all times. Figure 7 shows optimal energy-voltage points in both customized and generic platforms. Each point shows the maximum amount of energy generated, while satisfying a specific voltage value for at least $60 \%$ of the time. Therefore each of the found voltage/energy optimal points can be chosen based on different design parameters such as battery specification and application needs or user defined constraints. The result of the optimal location of a generator for a generic platform that produces maximal energy savings over all individuals is depicted in Figure 8.

\section{CONCLUSIONS}

We developed an optimization intensive approach for energy harvesting in medical shoes. We used readings from 64 pressure sensors of 30 subjects. Using this information and a proposed scenario approach we found size and location for placement of piezoelectric transducer in both common and customized platforms, optimizing for maximal amount of collected energy and maximal amount of collected energy with a specified voltage achieved.

\section{REFERENCES}

[1] S. Roundy, P.K. Wright, J.M. Rabaey, Energy Scavenging for Wireless Sensor Networks, Kluwer Academic Publishers, 2003.

[2] J.A. Paradiso and T. Starner, Energy scavenging for mobile and wireless electronics. IEEE Pervasive Comput., vol. 4, no. 1, pp. 18-27, 2005.

[3] A.D. Joseph, Energy harvesting projects, IEEE Perv. Comput., vol. 4, no. 1, pp. 69-71, Jan.-Mar. 2005.

[4] T. Liu, et. al, Development of a wearable sensor system for quantitative gait analysis, Measurement, vol. 42, no. 7, pp. 978-988, 2009.

[5] H. A. Sodano, et. al., Comparison of piezoelectric energy harvesting devices for recharging batteries J. Intell. Mater. Syst. Struct. 799-807.

[6] S. Priya, Advances in energy harvesting using low profile piezoelectric transducers, Journal of Electroceram., vol. 19, pp. 167-184, Sep. 2007.

[7] A. Martínez-Nova, et al, Biofoot ${ }^{\circledR}$ in-shoe system: normal values and assessment of the reliability and repeatability, Foot 17 (4) 2007, pp. 190-196.

[8] T. Wacharasindhu and J.W. Kwon, A micromachined energy harvester from a keyboard using combined electromagnetic and piezoelectric conversion, Journal of Micromechanics and Microeng., Vol 18, 2008.

[9] S.R. Anton, H.A. Sodano,A review of power harvesting using piezoelectric materials, Smart Mater. Struct., Vol. 16, No. 3, 2007.

[10] W. Ye, et. Al., Shoe-Mouse: An integrated intelligent shoe, Proc. IEEE/RSJ Int. Conf. Intell. Robots Syst. (IROS), pp 1163-1167, 2005.

[11] H.L.P. Hurkmans, et al, Accuracy and Repeatability of the Pedar Mobile System in Long-term Vertical Force Measurements, Gait and Posture, vol. 23, issue 1, pp. 118-125, 2006.

[12] J.A. Paradiso, et al., Design and implementation of expressive footware, IBM Systems Journal, vol. 39, no. 3, pp 511-519, 2000.

[13] M. Chen, B. Huang, Y. Xu, Intelligent shoes for abnormal gait detection, IEEE Int. Conf. Robot. Autom. (ICRA), pp. 2019-2024, 2009.

[14] Pedar: plantar pressure system. http://www.novel.de.

[15] F-Scan ${ }^{\circledR} \mathrm{http}: / / \mathrm{www} \cdot$ tekscan.com/medical/system-fscan1.html.

[16] S. Patel, et al, Monitoring Motor Fluctuations in Patients With Parkinson's Disease Using Wearable Sensors, IEEE Transactions on Information Technology in Biomedicine, Nov, Volume 13, 2009.

[17] Myung-kyung Suh, et. al., Optimizing Interval Training Protocols Using Data Mining Decision Trees, BSN'09, pp. 318-323.

[18] N.S. Shenck and J.A. Paradiso, Energy scavenging with shoe-mounted piezoelectrics, Micro, IEEE, vol.21, no.3, pp. 30-42, May/Jun 2001.

[19] H. Noshadi, et al., Energy Optimization in Wireless Medical Systems Using Physiological Behavior, ACM/BMES Wireless Health, pp. 128136,2010 . 